\title{
Access to HIV/AIDS care: a systematic review of socio-cultural determinants in low and high income countries
}

Sara Gari ${ }^{1,2}$, Camilo Doig-Acuña ${ }^{3}$, Tino Smail ${ }^{4}$, Jacob RS Malungo ${ }^{5}$, Adriane Martin-Hilber ${ }^{1,2}$ and Sonja Merten ${ }^{1,2^{*}}$

\begin{abstract}
Background: The role of socio-cultural factors in influencing access to HIV/AIDS treatment, care and support is increasingly recognized by researchers, international donors and policy makers. Although many of them have been identified through qualitative studies, the evidence gathered by quantitative studies has not been systematically analysed. To fill this knowledge gap, we did a systematic review of quantitative studies comparing surveys done in high and low income countries to assess the extent to which socio-cultural determinants of access, identified through qualitative studies, have been addressed in epidemiological survey studies.

Methods: Ten electronic databases were searched (Cinahl, EMBASE, ISI Web of Science, IBSS, JSTOR, MedLine, Psyinfo, Psyindex and Cochrane). Two independent reviewers selected eligible publications based on the inclusion/exclusion criteria. Meta-analysis was used to synthesize data comparing studies between low and high income countries.
\end{abstract}

Results: Thirty-four studies were included in the final review, 21 (62\%) done in high income countries and 13 (38\%) in low income countries. In low income settings, epidemiological research on access to HIV/AIDS services focused on socio-economic and health system factors while in high income countries the focus was on medical and psychosocial factors. These differences depict the perceived different barriers in the two regions. Common factors between the two regions were also found to affect HIV testing, including stigma, high risk sexual behaviours such as multiple sexual partners and not using condoms, and alcohol abuse. On the other hand, having experienced previous illness or other health conditions and good family communication was associated with adherence to ART uptake. Due to insufficient consistent data, a meta-analysis was only possible on adherence to treatment.

Conclusions: This review offers evidence of the current challenges for interdisciplinary work in epidemiology and public health. Quantitative studies did not systematically address in their surveys important factors identified in qualitative studies as playing a critical role on the access to HIV/AIDS services. The evidences suggest that the problem lies in the exclusion of the qualitative information during the questionnaire design. With the changing face of the epidemic, we need a new and improved research strategy that integrates the results of qualitative studies into quantitative surveys.

Keywords: Socio-cultural barriers, Access, Adherence, HIV/AIDS, Antiretroviral therapy, Survey study, Systematic review

\footnotetext{
*Correspondence: sonja.merten@unibas.ch

${ }^{1}$ Department of Epidemiology and Public Health, Swiss Tropical and Public Health Institute, Basel, Switzerland

${ }^{2}$ University of Basel, Basel, Switzerland

Full list of author information is available at the end of the article
} 


\section{Background}

Socio-cultural diversity needs to be considered during the design of HIV/AIDS policies and programmes. Social attitudes and prejudices towards people living with HIV/ AIDS, sexual taboos and gender inequality are some of the most important challenges for prevention and treatment of HIV/AIDS [1-6]. Despite an improved performance of health and community services, people living with HIV/ AIDS continue to face persistent, deeply rooted, social and cultural barriers.

International donors, public health experts, programme planners and policy makers need to begin to recognize the need to take into account this socio-cultural diversity in program planning. Hence it is essential to gather the scientific evidence generated so far on this topic. Much of this evidence has been generated from qualitative studies. The most important and frequently reported socio-cultural barriers in both low income and high income countries include fear of disclosure, anticipation of stigma, limited social support, interpersonal violence and alcohol abuse [2-7]. To better understand the distribution, frequency, and potential impact that these factors may have on the population, quantitative epidemiological surveys should ideally incorporate similar questions. It is currently unclear to what extent socio-cultural determinants of access, identified by qualitative studies, are addressed in survey studies. To our knowledge there is no systematic review of epidemiological literature available to clarify this question. Therefore, this article seeks to answer three questions: What socio-cultural factors have been measured in epidemiologic studies to assess access to HIV/AIDS services? What are the differences between factors measured in low and high income countries? And what are the associations and effect sizes of these factors?

\section{Methods}

A systematic search of quantitative studies addressing factors that influence access to HIV testing, uptake of antiretroviral therapy (ART) and adherence to antiretroviral (ARV) regimens was performed. In order to determine the differences between the factors studied in low and high income and test whether these differences were consistent with the findings of qualitative studies for each context we scrutinized studies from low and low-middle income countries and from upper-middle and high-income countries as defined by the World Bank Classification [8].

To facilitate the comparative analysis and the description of the results the countries belonging to these four income groups were reclassified into two broader categories: low and high income countries. The category low-income countries included low income countries and low-middle income countries and the high-income category included upper-middle and high-income countries.

\section{Search strategy}

The search was restricted to studies with sample population over 18 years old and in English, French, German, Spanish, Portuguese and Italian. No other limitations were applied. The systematic search lasted one day with date $07^{\text {th }}$ October 2011. The search terms were: 'HIV OR AIDS', 'voluntary counselling and testing' 'HAART OR antiretroviral",' 'compliance OR adherence,' 'factors OR determinant" OR barriers' and 'motivat" OR facilitat"'. The search included Cinahl, EMBASE, CSA databases, IBSS, ISI Web of Science, JSTOR, MedLine, Psyinfo and Psyindex and the Cochrane Database of Systematic Reviews. Conference abstracts from the International AIDS Society conferences web site were also searched. We complemented the search by reviewing the bibliographies of key papers. The detailed search strategy is available upon request.

\section{Inclusion and exclusion criteria}

To be eligible, articles needed to: report an original research study; measure one of these three outcomes: HIV testing, initiation of ART and adherence to antiretroviral therapy; study associations (of one of the three outcomes) with socio-cultural factors; target adult participants over 18 years old; apply a survey methodology for data collection; estimate risk effects as an outcome; and control for confounding in the analysis. Studies that reported socio-demographic or socio-economic factors but not any of the other categories of socio-cultural variables were excluded.

\section{Study selection and quality appraisal}

The study selection followed a four-step process: title review; abstract review; full text review and quality appraisal. First, two of the authors independently reviewed all identified study titles. Duplicates and titles that did not meet the inclusion criteria were removed. The same authors then independently assessed the abstracts, and then the full papers of those abstracts that met the eligibility criteria. Finally, a quality appraisal was done on all full texts using consolidated criteria of the STROBE guidelines [9]. STROBE is a checklist of 22 items that must be addressed in the report of observational studies. This list is not really a tool to assess the quality of observational research but provides valuable guidance on the quality of reporting the studies.

In addition, a modified version of the Newcastle-Ottawa Scale (NOS) for observational studies (e.g. cross-sectional and cohort studies) was used to assess the methodological quality [10]. NOS is a tool to assess the quality of non randomized studies to be used in a systematic review. Each study is judged with a 'star system' on three points: the selection of the study groups, the comparability of the groups, and the ascertainment of the exposure or outcome. In our review, only studies in which five of nine items on the NOS 
were deemed satisfactory and in which appropriate statistical analysis (e.g. multivariate controlling for confounders) was conducted were considered to be of high methodological quality (maximum score of 9). At each stage of the quality assessment the reviewers discussed together until a consensus on which studies to include was reached. Finally, the reviewers manually searched the reference lists of the included articles for further key studies that could potentially be included in the analysis.

\section{Data extraction and classification}

The following data was extracted and summarized in evidence tables: citation; year of publication; country; study design and sampling; characteristics of the study population; community versus facility based; sample; outcomes (HIV testing, uptake of ART, adherence and dropout); and factors that facilitated and/or hindered access to HIV care such as: socio-demographic; socioeconomic; medical; health system; knowledge and beliefs; risky health behaviours; psychosocial; stigma and discrimination; family and interpersonal violence; communication about HIV/AIDS; community prevalence. An overview of data extraction is provided in Tables 1 and 2 .

The data was extracted and reviewed in duplicate from identified studies. Common indicators were grouped into bigger categories (factors) in duplicate by independent reviewers. Disagreements in the categorization of the factors were discussed until consensus was reached. Countries of the study were classified as high or low income countries as defined by the World Bank [8]. Odds ratio (OR) or similar estimates (e.g. relative risk, hazard ratio) and their respective confidence intervals for every unique risk estimate involving a specific indicator and the uptake of testing, initiating ART and adhering to ART were extracted when available.

\section{Statistical analysis}

Descriptive statistics were used to examine patterns across countries with respect to: proportion (\%) of factors studied in relation to access to HIV/AIDS care, estimated effect sizes (adjusted odd ratios) identified (protective $v s$ risk) for each factor and the precision around the estimates ( $95 \%$ confidence intervals). Additionally meta-analysis was done for nine specific socio-cultural factors identified by the studies as statistically significantly associated with adherence to antiretroviral therapy. Despite the expected heterogeneity within the review (great variability of the measures used to study socio-cultural factors) we assessed the comparability of the results from individual studies using the $\mathrm{I}^{2}$ statistic for quantifying inconsistency. An overall $\mathrm{I}^{2}$ test-value greater than $60 \%$ was considered as indicative of a high level of heterogeneity for which statistical pooling was not appropriate. Further analyses included sensitivity analysis performed by removing the studies that contributed to the heterogeneity in the meta-analysis and subgroup analyses to compare high-income countries with low-income countries. A p-value of less than 0.05 was considered statistically significant. Analyses were performed in STATA 12.1.

\section{Results \\ Study selection}

The primary search strategy identified 1,671 potentially relevant citations. After searching for duplicates 715 citations were discarded. Initial title and abstract screening excluded 815 manuscripts based on the inclusion - exclusion criteria. The remaining 141 were then retrieved for full text review. A further 86 manuscripts were excluded as not meeting the inclusion criteria. The remaining 55 manuscripts were quality appraised and 21 were excluded as they did not deal with confounding in their analysis. Thirtyfour articles were included in our analysis. Figure 1 displays the flow chart of the selection process.

\section{Study characteristics}

All 34 included studies employed a quantitative methodology (surveys) and used structured questionnaires to determine potential factors. 13 studies (38\%) were conducted in low income countries [11-23] and 21 (62\%) in high income countries [24-44]. The studies conducted in low income countries included one from Cameroon [11], Zambia [12], Nigeria [13], Peru [14], Ethiopia [15], Kenya [16], Ghana [17], Uganda [18], Vietnam [19], India [20], Tanzania [21], Zimbabwe [22] and Malawi [23]. From high income countries, nine studies were from USA [26,28,30,33,34,39-42], four from South Africa [31,35,36,44], three from Brazil $[27,37,38]$ and one each from Italy [24], Denmark [25], Australia [29], Thailand [32] and China [43].

A total of 29 studies (85\%) were clinic based [11-16,18-21,24-35,37-40,42] and five (15\%) were situated at community level $[17,22,23,36,44]$. Twenty-four studies (70\%) focused on adherence to ART [11-15,20,21, 24-30,32-39,41-43], five studies (15\%) focused on uptake of voluntary and counselling testing (VCT) [17,22,23,36,44], four $(11 \%)$ on ART initiation $[16,18,19,26]$ and one $(3 \%)$ on attrition [31]. Table 1 displays the characteristics of the studies.

\section{Factors measured by quantitative surveys to study access} to HIV/AIDS-care in high- and low-income countries

12 factors were identified as measured by the studies to assess access to HIV care: (i) socio-demographic, (ii) socioeconomic, (iii) medical, (iv) health system, (v) knowledge and beliefs, (vi) risky health behaviours, (vii) psychosocial, (viii) stigma and discrimination, (ix) family (x) interpersonal violence, (xi) communication about HIV/AIDS and (xii) community prevalence. Table 2 shows an outline of the factors identified per study. 
Table 1 Characteristics of the studies

\begin{tabular}{|c|c|c|c|c|c|c|c|}
\hline Source & Year & Country & Design & Population & Setting & $\mathrm{N}$ & Outcome \\
\hline Aloisis & 2002 & Italy & Longitudinal & HIV + adults & Clinic & 366 & Adherence \\
\hline Bardford & 2005 & Denmark & Cross sectional & HIV + adults & Clinic & 887 & Adherence \\
\hline Boyer & 2011 & Cameroon & Cross sectional & HIV + adults & Clinic & 2,381 & Adherence \\
\hline Carlucci & 2008 & Zambia & Cross sectional & HIV + adults & clinic & 424 & Adherence \\
\hline Charurat & 2010 & Nigeria & Longitudinal & HIV + adults & Clinic & 4,529 & Adherence \\
\hline Cunningham & 1999 & USA & Longitudinal & HIV + adults & Clinic & 2,864 & Initiation ART \\
\hline de Castilho & 2006 & Brazil & Longitudinal & HIV + adults & Clinic & 498 & Adherence \\
\hline Franke & 2011 & Peru & Longitudinal & HIV + adults & Clinic & 132 & Adherence \\
\hline Gebo & 2003 & USA & Cross sectional & HIV + adults & Clinic & 196 & Adherence \\
\hline Giday & 2010 & Ethiopia & Cross sectional & HIV + adults & Clinic & 510 & Adherence \\
\hline Grierson & 2011 & Australia & Cross sectional & HIV + adults & Clinic & 1,106 & Adherence \\
\hline Holmes & 2007 & USA & Longitudinal & HIV + adults & Clinic & 116 & Adherence \\
\hline Karcher & 2007 & Kenya & Longitudinal & HIV + adults & Clinic & 159 & Initiation ART \\
\hline Koku & 2011 & Ghana & Cross sectional & Female 15-49 & Community & 3,766 & HIV testing \\
\hline Kranzer & 2010 & South Africa & Longitudinal & HIV + adults & Clinic & 1,154 & Defaulting \\
\hline Kranzer_b & 2008 & Malawi & Cross sectional & People 18-59 & Community & 2,047 & HIV testing \\
\hline $\mathrm{Li}$ & 2010 & Thailand & Cross sectional & HIV + adults & Clinic & 386 & Adherence \\
\hline MacPhail & 2009 & South Africa & Cross sectional & People 15-24 & Community & 7,655 & HIV testing \\
\hline Martinez & 2008 & Uganda & Cross sectional & HIV + adults & Clinic & 421 & Initiation ART \\
\hline Mugavero & 2006 & USA & Cross sectional & HIV + adults & Clinic & 611 & Adherence \\
\hline $\mathrm{Nam}$ & 2010 & Vietnam & Cross sectional & HIV + adults & Clinic & 353 & Initiation ART \\
\hline Okonsy & 2011 & USA & Cross sectional & HIV + adults & Clinic & 558 & Adherence \\
\hline Peltzer & 2011 & South Africa & Cross sectional & HIV + adults & Clinic & 735 & Adherence \\
\hline Pettifor & 2010 & South Africa & Cross sectional & People over 15 years old & Community & 198 & HIV testing \\
\hline Pinheiro & 2002 & Brazil & Cross sectional & HIV + adults & Clinic & 195 & Adherence \\
\hline Remien & 2007 & Brazil & Cross sectional & HIV + adults & Clinic & 200 & Adherence \\
\hline Rintamaki & 2006 & USA & Cross sectional & HIV + adults & Clinic & 204 & Adherence \\
\hline Sambisa & 2010 & Zimbabwe & Cross sectional & People over 15 years old & Community & 12,254 & HIV testing \\
\hline Sarna & 2008 & India & Cross sectional & HIV + adults & Clinic & 310 & Adherence \\
\hline Sayles & 2006 & USA & Longitudinal & HIV + adults & Clinic & 1,910 & Adherence \\
\hline Sayles & 2009 & USA & Cross sectional & HIV + adults & Clinic & 202 & Adherence \\
\hline Van Servellen & 2005 & USA & Cross sectional & HIV + adults & Clinic & 85 & Adherence \\
\hline Wang & 2007 & China & Cross sectional & HIV + adults & Clinic & 181 & Adherence \\
\hline Watt & 2009 & Tanzania & Cross sectional & HIV + adults & Clinic & 340 & Adherence \\
\hline
\end{tabular}

Comparative analysis showed important divergences across countries. Surveys in low income countries tended to focus on the study of socio-demographic, socioeconomic and health system factors in relation to access to HIV/AIDS services [24,25,27,29,31,32,34-40,42-44] while in high income countries the emphasis was on medical and psychosocial conditions [25-27,29,32,33,35,38,40-42]. Figure 2 graphically displays the distribution of factors by country. Socio-demographic, economic, clinical and sexual behavioural factors were considerably measured in all surveys conducted in low and high income countries while interpersonal relationships, communication and interpersonal violence factors remained highly understudied in both low and high economic countries. Table 3 displays the ranking of factors by the proportion of studies where they were included.

\section{Reported risk factors significantly associated with access to HIV/AIDS services across countries}

Non-uptake of HIV testing in high income countries was associated with socio-demographic constructs such as being black [44], being between 25 to 34 years old, and living in a community with high HIV testing refusal rates [23]. Other barriers included high risk sexual behaviours [36] such as multiple sexual partners and not using condoms and enacted stigma [39]. In low income countries, the 
Table 2 Outline of the factors identified per study

\begin{tabular}{|c|c|c|c|c|c|c|c|c|c|c|c|c|}
\hline Country group & Source & Clinical & Disclosure & Location & Violence & $\begin{array}{c}\text { Risk } \\
\text { behavior }\end{array}$ & $\begin{array}{l}\text { Health } \\
\text { system }\end{array}$ & $\begin{array}{l}\text { Health } \\
\text { Beliefs }\end{array}$ & Psychosocial & $\begin{array}{c}\text { Social } \\
\text { support }\end{array}$ & SES & Stigma \\
\hline \multirow{13}{*}{$\begin{array}{l}\text { Low and low } \\
\text { middle income } \\
\text { countries }\end{array}$} & Boyer & $x$ & . & . & . & $x$ & $x$ & . & . & $x$ & $x$ & $x$ \\
\hline & Carlucci & $x$ & . & . & . & & $x$ & . & . & . & . & $x$ \\
\hline & Charurat & $x$ & $x$ & . & . & . & $x$ & . & . & . & $x$ & . \\
\hline & Franke & $x$ & . & . & . & $x$ & . & . & $x$ & $x$ & $x$ & $x$ \\
\hline & Giday & $x$ & . & . & . & $x$ & . & . & . & $x$ & $x$ & . \\
\hline & Karcher & $x$ & . & . & . & . & . & . & . & . & . & . \\
\hline & Koku & . & . & . & . & $x$ & . & $x$ & $x$ & . & $x$ & $x$ \\
\hline & Martinez & $x$ & . & . & . & $x$ & . & . & $x$ & . & . & . \\
\hline & $\mathrm{Nam}$ & $x$ & $x$ & . & . & $x$ & $x$ & . & . & $x$ & $x$ & . \\
\hline & Sarna & $x$ & . & . & . & . & $x$ & . & $x$ & . & $x$ & . \\
\hline & Watt & . & . & . & . & . & $x$ & . & $x$ & . & . & . \\
\hline & Kranzer_b & . & . & $x$ & . & . & . & . & . & . & $x$ & . \\
\hline & Sambisa & . & . & . & . & $x$ & . & $x$ & . & . & $x$ & $x$ \\
\hline \multirow{21}{*}{$\begin{array}{l}\text { High and upper } \\
\text { middle income } \\
\text { countries }\end{array}$} & Aloisis & $x$ & . & . & . & $x$ & . & . & . & . & . & . \\
\hline & Bardford & $x$ & . & . & . & $x$ & $x$ & . & $x$ & $x$ & . & . \\
\hline & Cunningham & . & . & . & . & . & . & . & $x$ & . & $x$ & . \\
\hline & De Castilho & $x$ & . & . & . & $x$ & & . & $x$ & & & . \\
\hline & Gebo & . & . & . & . & $x$ & . & $x$ & . & $x$ & $x$ & . \\
\hline & Grierson & $x$ & . & . & . & $x$ & $x$ & $x$ & $x$ & . & . & $x$ \\
\hline & Holmes & . & . & . & . & $x$ & . & . & . & . & $x$ & . \\
\hline & Kranzer & $x$ & . & . & . & . & . & . & . & . & . & . \\
\hline & $\mathrm{Li}$ & $x$ & $x$ & . & . & 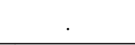 & $x$ & . & $x$ & $x$ & $x$ & $x$ \\
\hline & Mugavero & . & . & . & . & $x$ & . & . & $x$ & . & $x$ & . \\
\hline & Okonsy & $x$ & . & . & . & . & . & . & . & $x$ & . & . \\
\hline & Peltzer & $x$ & . & . & . & $x$ & . & . & $x$ & $x$ & $x$ & $x$ \\
\hline & Pettifor & $x$ & $x$ & . & . & . & $x$ & $x$ & . & . & . & $x$ \\
\hline & Pinheiro & $x$ & . & . & . & . & . & . & 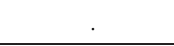 & . & $x$ & . \\
\hline & Remien & $x$ & . & . & . & . & . & . & $x$ & . & . & . \\
\hline & Rintamaki & $x$ & . & . & . & . & . & . & - & . & . & $x$ \\
\hline & Sayles_a & . & . & . & . & . & . & . & $x$ & . & . & $x$ \\
\hline & Sayles_b & $x$ & . & . & $x$ & $x$ & . & . & $x$ & . & $x$ & . \\
\hline & Vanservellen & $x$ & . & . & . & . & $x$ & $x$ & $x$ & $x$ & & . \\
\hline & Wang & $x$ & . & . & . & . & $x$ & . & . & . & . & . \\
\hline & Mc Phail & $x$ & $x$ & . & . & $x$ & & $x$ & & & & $x$ \\
\hline
\end{tabular}

barriers associated to HIV testing included belonging to the age group from 25 to over 45 [17,22], having unprotected sex and having sex with a non-spousal or non-cohabiting partner [17] and anticipated stigma [17,22].

Non-uptake of ART in high income countries was only statistically significantly associated with having other competing subsistence needs [26]. In low income countries, the barriers to initiate ART included indirect costs of health care, not having a known HIV-positive family member, non-disclosure of HIV status and having additional pregnancies $[11,19,20]$.
In high income countries, low adherence to ART was associated with being black [41,44], being between 25-34 years old $[27,41]$ and having less than primary education [27]. Other barriers were clinical and treatment factors such as protease inhibitor ART regimens [34], frequent doses of ART [29,37], experience of side effects [34,38], feeling unhappy with the treatment [25], initiating the treatment with a CD4 count over 200 Cells/mL3 [31]. Alcohol and/or substance abuse [24,27-29,33] and anticipated and internalized stigma were associated with low levels of adherence [29,33,35,39]. 


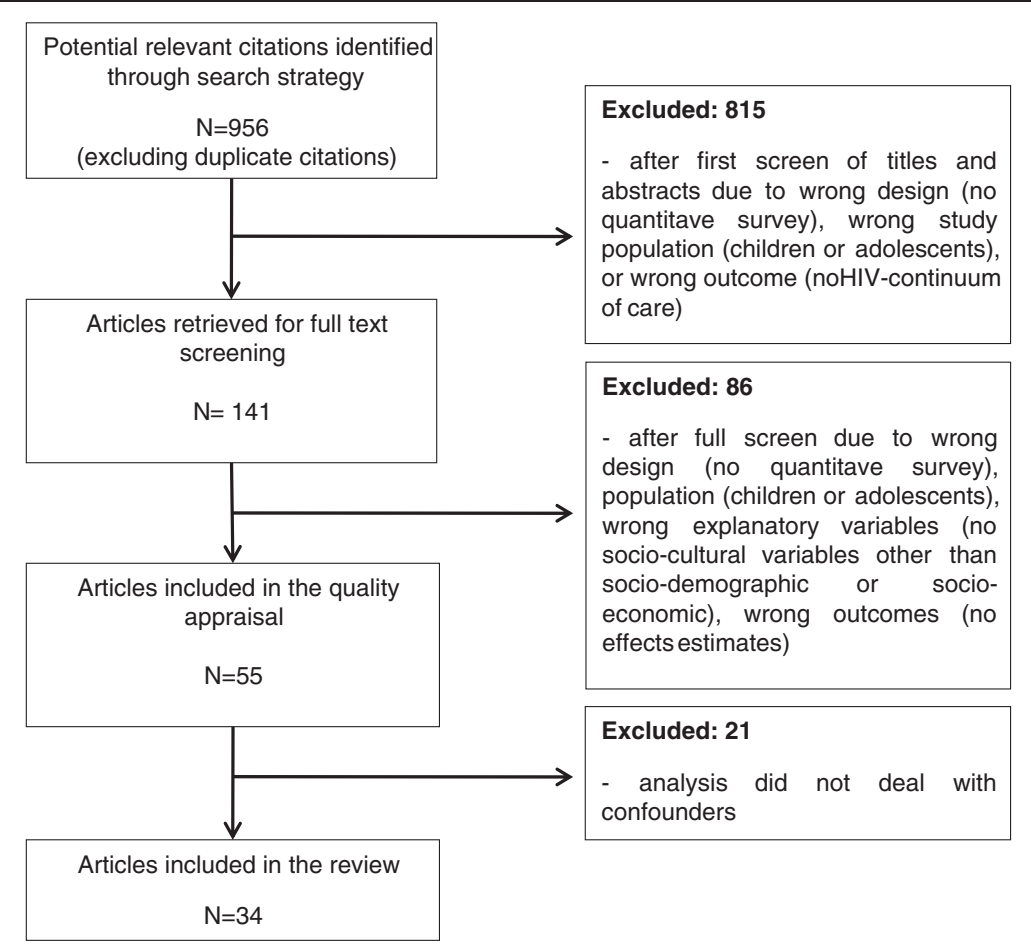

Figure 1 Flow chart.

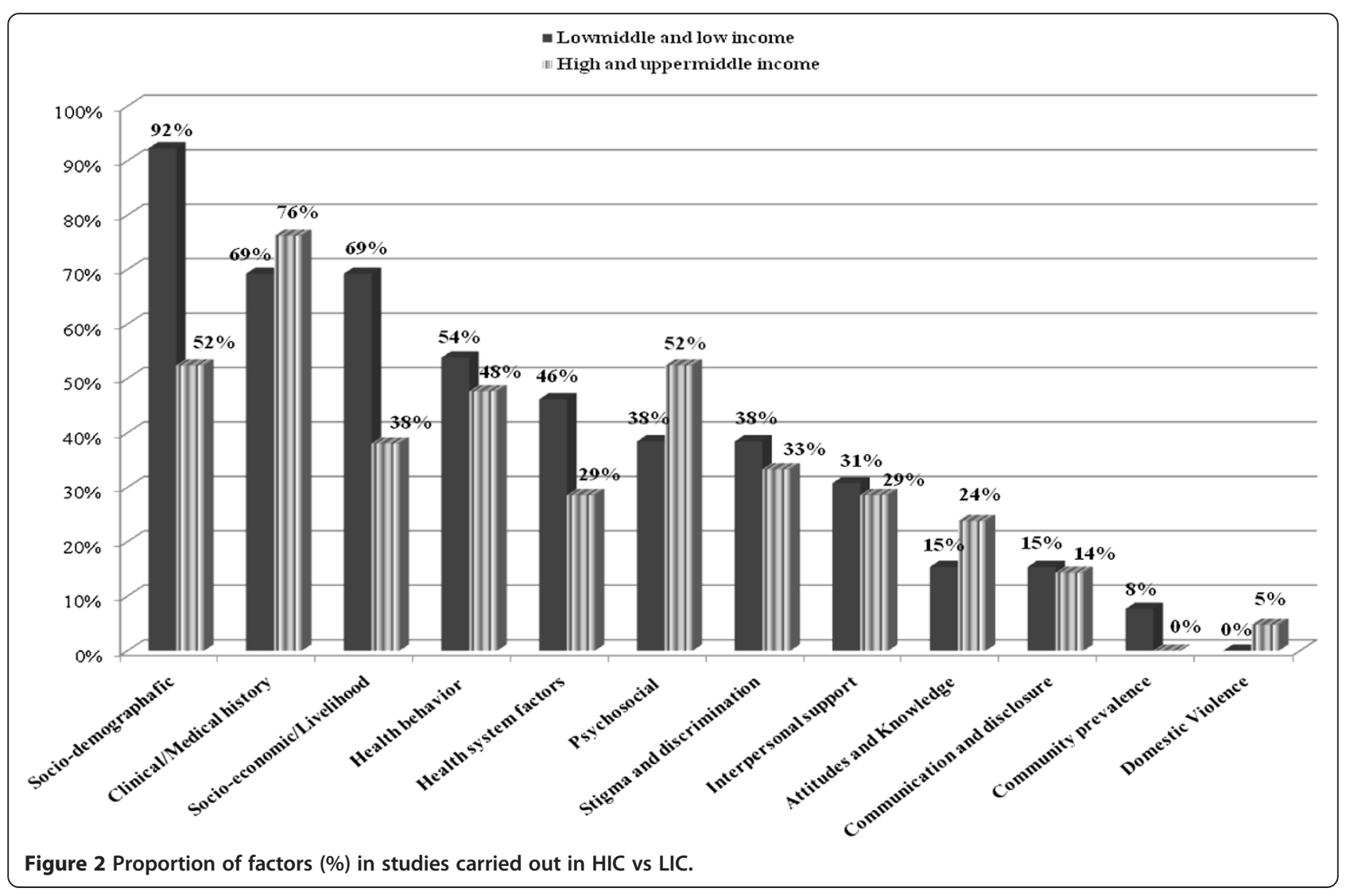


Table 3 Ranking of proportion (\%) of factors studied in low and high income countries

\begin{tabular}{|c|c|c|c|c|c|}
\hline \multicolumn{3}{|c|}{ Low income countries } & \multicolumn{3}{|c|}{ High income countries } \\
\hline Rank & & $\%$ & Rank & & $\%$ \\
\hline 1 & Socio-demographic factors & $92 \%$ & 1 & Clinical factors & $76 \%$ \\
\hline 2 & Clinical factors & $69 \%$ & 2 & Socio-demographic factors & $52 \%$ \\
\hline 3 & Socio-economic factors & $69 \%$ & 3 & Psychosocial factors & $52 \%$ \\
\hline 4 & Health behavior factors & $54 \%$ & 4 & Health behavior factors & $48 \%$ \\
\hline 5 & Health system factors & $46 \%$ & 5 & Socio-economic factors & $38 \%$ \\
\hline 6 & Psychosocial factors & $38 \%$ & 6 & Stigma and discrimination & $33 \%$ \\
\hline 7 & Stigma and discrimination & $38 \%$ & 7 & Health system factors & $29 \%$ \\
\hline 8 & Social and family support & $31 \%$ & 8 & Social and family support & $29 \%$ \\
\hline 9 & Knowledge and beliefs & $15 \%$ & 9 & Knowledge and beliefs & $24 \%$ \\
\hline 10 & Communication and disclosure & $15 \%$ & 10 & Communication and disclosure & $14 \%$ \\
\hline 11 & Community characteristics & $8 \%$ & 11 & Domestic Violence & $5 \%$ \\
\hline 12 & Domestic Violence & $0 \%$ & 12 & Community characteristics & $0 \%$ \\
\hline
\end{tabular}

In low income countries, age groups associated with low adherence to ART were 19-30 years and over 50 years [21]. The latter deviate from the risk age reported from high income countries where being over 50 was found to be protective. Other risk factors included having a main couple but not living together [11], being Muslim [13], being female [14], food insufficiency [14] and household financial problems $[11,15]$. Alcohol abuse and experienced discrimination were associated with low adherence also in low income countries [11]. With respect to clinical and treatment factors in low income countries initiation of ART with CD4 over 200 cells/ml3, being on ART less than 15 months [13,15] and having switched ART regimen [11] were associated with low adherence. Health system barriers included ARV stock outs, inadequate communication with health staff $[11,13,21]$ and health care indirect costs $[11,19,20]$. One study reported an inverse association between free cost of ARV and adherence [20]. Concerning interpersonal and psychosocial factors, perceived lack of family support [11], not having disclosed to the family members [19] and feeling depressed [20] were negatively associated with adherence.

\section{Reported protective factors significantly associated with access to HIV/AIDS services across countries}

In high income countries, protective factors for uptake of HIV testing were being female [36,44], having a history of previous illnesses $[31,34,41,44]$, having disclosed and/or having conversations about HIV/AIDS with parents [44] and believe that most people do not want to get tested for HIV or do it only if they are sick $[36,44]$. In low income countries, being educated beyond primary school [17], single [22], affiliated to non Christian religions [17], living in a high prevalence community [22], knowing someone infected with HIV/AIDS [22], practicing safer sexual behaviours such as using condoms and being exposed to media $[17,22]$ were positively associated with uptake of testing.

In low income countries, belonging to the age group 30 to 40 years of age and having greater than a primary school education facilitated initiation of ART [18]. Not drinking alcohol in the past year was also a protective factor [22]. No protective effects were reported from high income countries.

In high income countries, being older than 50 years of age [29] and on ART treatment for more than two years were positively associated with adherence to ART [35]. Previous illnesses or having other health conditions were positively associated with good adherence [31,34,41,44]. Self-perception of good health status [35,38] and no consumption of alcohol in the past year [30] were also protective. Good family communication [32,42], higher levels of treatment information [35] and believing in the benefits of ARVs [28] were facilitators of adherence. In low income countries, good social support and self-efficacy were positively associated with adherence as well as having disclosed to at least one family member about one's positive HIV status [13,14]. Experiencing other health conditions was also associated with a protective effect on adherence $[31,35,41,44]$.

No studies reported significant risk effects for defaulting.

\section{Combined effect sizes associated with adherence.}

The pool estimates of the 34 studies which included socio-cultural factors are shown for a) general sociodemographic factors, and b) specific socio-cultural factors. Due to an insufficient number of studies for other outcomes, only adherence could be included in the model.

The meta-analysis showed that being male was associated with optimal adherence in low income countries $(\mathrm{OR}=0.16,95 \% \mathrm{CI}=0.04-0.66)$ while the association with being female was not statistically significant. Conversely, 
Table 4 Meta-estimates: effect of socio-demographic factors on adherence to ART

\begin{tabular}{|c|c|c|c|c|c|}
\hline Socio-demographic & Pool ES & $\mathrm{Cl} 95 \%$ & p value* & Pool studies & $I^{2}$ \\
\hline Male & 0.77 & $0.48-1.24$ & 0.28 & 7 & $61.3 \%$ \\
\hline High income countries & 0.94 & $0.57-1.55$ & 0.81 & 4 & $54.6 \%$ \\
\hline Low income countries & 0.16 & $0.04-0.66$ & 0.01 & 3 & $0.0 \%$ \\
\hline Female & 1.05 & $0.97-1.13$ & 0.27 & 7 & $49.1 \%$ \\
\hline High income countries & 1.29 & $1.06-1.58$ & 0.01 & 4 & $34.6 \%$ \\
\hline Low income countries & 0.99 & $0.91-1.08$ & 0.85 & 3 & $0.0 \%$ \\
\hline Married & 1.10 & $0.85-1.42$ & 0.46 & 2 & $0.0 \%$ \\
\hline High income countries & 1.10 & $0.84-1.45$ & 0.49 & 1 & - \\
\hline Low income countries & 1.10 & $0.56-2.16$ & 0.78 & 1 & - \\
\hline Single & 2.49 & $1.51-4.12$ & 0.00 & 3 & $0.0 \%$ \\
\hline High income countries & 1.53 & $0.42-5.56$ & 0.52 & 1 & - \\
\hline Low income countries & 2.72 & $1.58-4.69$ & 0.00 & 2 & - \\
\hline Separated/divorced & - & - & - & - & - \\
\hline High income countries & - & - & - & - & - \\
\hline Low income countries & 1.07 & $0.42-2.73$ & 0.89 & 2 & $0.0 \%$ \\
\hline Age less than 20 & 1.14 & $0.96-1.37$ & 0.14 & 2 & $60.1 \%$ \\
\hline High income countries & 0.47 & $0.96-1.37$ & 0.13 & 1 & - \\
\hline Low income countries & 1.18 & $0.98-1.42$ & 0.08 & 1 & - \\
\hline Age less than 30 & 1.04 & $1.01-1.07$ & 0.01 & 2 & $0.0 \%$ \\
\hline High income countries & - & - & - & - & - \\
\hline Low income countries & 1.04 & $1.01-1.07$ & 0.01 & 2 & $0.0 \%$ \\
\hline Age 20-30 & 1.38 & $1.21-1.58$ & 0.00 & 5 & $60.2 \%$ \\
\hline High income countries & 1.30 & $0.86-1.94$ & 0.21 & 3 & $20.2 \%$ \\
\hline Low income countries & 1.39 & $1.21-1.60$ & 0.00 & 2 & $74.0 \%$ \\
\hline Age $30-50$ & 1.08 & $0.97-1.19$ & 0.16 & 6 & $0.0 \%$ \\
\hline High income countries & 1.06 & $0.77-1.46$ & 0.72 & 4 & $0.2 \%$ \\
\hline Low income countries & 1.08 & $0.97-1.20$ & 0.17 & 2 & $0.0 \%$ \\
\hline Age over 50 years & 0.80 & $0.59-1.08$ & 0.14 & 5 & $59.4 \%$ \\
\hline High income countries & 0.55 & $0.41-0.75$ & 0.00 & 4 & $0.0 \%$ \\
\hline Low income countries & 6.68 & $3.15-14.15$ & 0.00 & 1 & - \\
\hline No education & 1.76 & $1.19-2.60$ & 0.01 & 3 & $0.0 \%$ \\
\hline High income countries & 1.69 & $0.82-3.48$ & 0.16 & 1 & - \\
\hline Low income countries & 1.78 & $1.12-2.85$ & 0.02 & 2 & $0.0 \%$ \\
\hline Primary education & 0.98 & $0.85-1.13$ & 0.80 & 4 & $9.3 \%$ \\
\hline High income countries & 0.99 & $0.86-1.15$ & 0.91 & 3 & $0.0 \%$ \\
\hline Low income countries & 0.30 & $0.06-1.49$ & 0.14 & 1 & - \\
\hline Secondary education & 1.04 & $0.95-1.14$ & 0.43 & 10 & $60.4 \%$ \\
\hline High income countries & 1.03 & $0.95-1.28$ & 0.75 & 6 & $73.7 \%$ \\
\hline Low income countries & 1.04 & $0.94-1.15$ & 0.47 & 4 & $19.8 \%$ \\
\hline Tertiary education & 0.85 & $0.58-1.24$ & 0.39 & 3 & $60.9 \%$ \\
\hline High income countries & 0.69 & $0.45-1.07$ & 0.10 & 2 & $44.8 \%$ \\
\hline Low income countries & 1.56 & $0.73-3.34$ & 0.25 & 1 & - \\
\hline
\end{tabular}

*Significance level $p<0.05$.

in high income countries low adherence was associated with females $(\mathrm{OR}=1.29,95 \% \mathrm{CI}: 1.06-1.58)$ while the association with male was not statistically significant. Being single $(\mathrm{OR}=2.72,95 \% \mathrm{CI}=1.58-4.69)$, and younger than $30(\mathrm{OR}=1.04,95 \% \mathrm{CI}=1.01-1.07)$ was significantly associated with lower adherence in low income countries. Being older than 50 years of age was associated with optimal adherence $(\mathrm{OR}=0.80,95 \% \mathrm{CI}=0.59-1.07)$ and was 
statistically significant in both settings; having no education was significantly associated with suboptimal adherence $(\mathrm{OR}=1.76,95 \% \mathrm{CI}=1.18-2.60)$ in both settings.

Socio-cultural factors associated with lower adherence included perceived lack of social support although sufficient data were available only in high income countries $(\mathrm{OR}=1.04,95 \% \mathrm{CI}: 1.01-1.07)$. Perceived social stigma had an overall risk factor $(\mathrm{OR}=2.17,95 \% \mathrm{CI}=1.52-3.09)$ in both settings. High risk health behaviours such as alcohol abuse $(\mathrm{OR}=1.75,95 \% \mathrm{CI}=1.41-2.18)$ and abuse drugs $(\mathrm{OR}=1.86,95 \% \mathrm{CI}=1.48-2.33)$ were also significant in both settings, while low levels of self-efficacy were negatively associated with adherence in both settings. This effect was stronger in high income countries $(\mathrm{OR}=2.1395 \% \mathrm{CI}=1.03-4.41)$ than in low income countries $(\mathrm{OR}=1.7595 \% \mathrm{CI}=1.91-1.31)$. Absence of symptoms of depression was positively associated with optimal adherence in both settings $(\mathrm{OR}=0.89,95 \% \mathrm{CI}=0.83-0.96)$. Tables 4 and 5 summarize the meta estimates of the sociodemographic and socio-cultural factors respectively.

\section{Discussion}

This review revealed a trend in quantitative survey studies to explore the same kinds of factors in relation to access to HIV/AIDS services. Overall the most studied factors in all regions, including Africa, Asia, Latin America and some groups and communities in North America, were socio-economic, medical and health risk behaviour. In low-income countries the research focus was on socioeconomic and health system factors while in high-income more attention was given to clinical and psychosocial factors such as depression, anxiety, self-efficacy and/or sexual identity. Socio-cultural factors including social and family support, interpersonal violence, and disclosure about HIV/AIDS received, in comparison, very little attention in both rich and poor countries.

These results should call the attention of survey researchers and systematic reviewers. The aforementioned socio-cultural factors have been widely published in qualitative studies [2-6] as critical factors that influence access to HIV/AIDS services, both in high and in low income countries. However most of the quantitative studies included in our review, from both high and low income countries, omitted them in their surveys or explored them very superficially. This is not justified as these factors are key issues for survey research. Our results suggest that the problem lied in the exclusion of qualitative information in the questionnaire design. Of the 34 studies included in this review, 27 [12-18,20,22-24,27,28,30-36,38-44] used validated measures from previous quantitative studies to derive their questionnaires and only seven studies, three, in low income countries [11,19,21] and four in high income countries $[25,26,29,37]$, conducted an informative phase, using qualitative methods, to inform the questionnaire development.

The exclusion of qualitative information during the questionnaire design in the rest of the studies could have led to over-emphasis in the research of the same kind of easily measured variables.

This compromises the interpretation and generalization of the evidence and its application to inform health policies

Table 5 Meta-estimates: effect of socio-cultural factors on adherence to ART

\begin{tabular}{|c|c|c|c|c|c|}
\hline Socio-cultural & Pool ES & $\mathrm{Cl} 95 \%$ & p value* & Pool studies & $1^{2}$ \\
\hline Low self-efficacy & 1.025 & $0.57-1.85$ & 0.94 & 3 & $63.1 \%$ \\
\hline High income countries & 2.13 & $1.03-4.41$ & 0.01 & 2 & $0.0 \%$ \\
\hline Low income countries & 1.75 & $1.91-1.31$ & 0.04 & 1 & - \\
\hline \multicolumn{6}{|l|}{ Lack of social support } \\
\hline High income countries & 1.04 & $1.01-1.08$ & 0.02 & 2 & $0.0 \%$ \\
\hline Low income countries & - & - & - & - & - \\
\hline No depression & 0.89 & $0.83-0.96$ & 0.00 & 2 & $0.0 \%$ \\
\hline High income countries & 0.92 & $0.82-1.04$ & 0.17 & 1 & - \\
\hline Low income countries & 0.88 & $0.80-0.96$ & 0.01 & 1 & - \\
\hline Stigma & 2.17 & $1.52-3.09$ & 0.00 & 2 & $62.1 \%$ \\
\hline High income countries & 3.70 & $1.92-7.42$ & 0.00 & 1 & - \\
\hline Low income countries & 1.74 & $1.14-2.65$ & 0.01 & 1 & - \\
\hline Abuse of alcohol & 1.75 & $1.41-2.18$ & 0.00 & 6 & $59.8 \%$ \\
\hline High income countries & 1.43 & $1.09-1.86$ & 0.01 & 4 & $24.0 \%$ \\
\hline Low income countries & 2.72 & 1.84-1.69 & 0.00 & 2 & $32.7 \%$ \\
\hline Abuse of drugs & 1.86 & $1.48-2.33$ & 0.01 & 9 & $1.1 \%$ \\
\hline High income countries & 1.89 & $1.49-2.41$ & 0.00 & 6 & $23.2 \%$ \\
\hline Low income countries & 1.58 & $0.78-3.22$ & 0.21 & 3 & $0.0 \%$ \\
\hline
\end{tabular}

*Significance level $p<0.05$. 
Table 6 Overview of measurement tools used to evaluate same socio-cultural constructs in different studies

\begin{tabular}{|c|c|c|c|c|}
\hline Source & Year & Country & Outcome & Measurement instrument \\
\hline \multicolumn{5}{|c|}{ Family support } \\
\hline Boyer & 2011 & LIC & Adherence & Self-reported \\
\hline Bardford & 2005 & $\mathrm{HIC}$ & Non adherence & Self-reported \\
\hline$\overline{\mathrm{Li}}$ & 2010 & $\mathrm{HIC}$ & Adherence & Adapted from FAD \\
\hline \multicolumn{5}{|c|}{ Social support } \\
\hline Vanservellen & 2005 & $\mathrm{HIC}$ & Adherence & MOS scale \\
\hline$\overline{\mathrm{Li}}$ & 2010 & $\mathrm{HIC}$ & Adherence & Adapted from MOS Scale \\
\hline Franke & 2011 & LIC & Adherence & Duke-UNC Functional Social Support Questionnaire \\
\hline Peltzer & 2010 & $\mathrm{HIC}$ & Adherence & Adapted Social Support Questionnaire \\
\hline Okonsy & 2011 & $\mathrm{HIC}$ & Adherence & Rates social support 1 to 10 \\
\hline Giday & 2010 & LIC & Adherence & Own questions \\
\hline \multicolumn{5}{|l|}{ Self-efficacy } \\
\hline Franke & 2011 & LIC & Adherence & ACTG \\
\hline Remien & 2007 & $\mathrm{HIC}$ & Adherence & ACTG \\
\hline Vanservellen & 2005 & $\mathrm{HIC}$ & Adherence & ACTG \\
\hline Watt & 2009 & LIC & Adherence & 10 item scale adapted \\
\hline \multicolumn{5}{|l|}{ Depression } \\
\hline Martinez & 2008 & LIC & Initiation ART & Hopkins Symptoms Checklist \\
\hline Franke & 2011 & LIC & Adherence & Hopkins Symptoms Checklist \\
\hline Peltzer & 2010 & $\mathrm{HIC}$ & Adherence & CES-D \\
\hline Vanservellen & 2005 & $\mathrm{HIC}$ & Adherence & CES-D \\
\hline Mugavero & 2006 & $\mathrm{HIC}$ & Adherence & $\mathrm{BSI}$ \\
\hline Sarna & 2008 & LIC & Adherence & $\mathrm{BDI}$ \\
\hline Bardford & 2005 & $\mathrm{HIC}$ & Non adherence & ACTG \\
\hline$\overline{\mathrm{Li}}$ & 2010 & $\mathrm{HIC}$ & Adherence & Thai Department of Mental Health \\
\hline Source & Year & Country & Outcome & Measurement instrument \\
\hline \multicolumn{5}{|c|}{ Patient-provider relationship } \\
\hline Bardford & 2005 & $\mathrm{HIC}$ & Non adherence & Self-reported \\
\hline Vanservellen & 2005 & $\mathrm{HIC}$ & Adherence & Satisfaction survey \\
\hline Watt & 2009 & LIC & Adherence & 9 item scale adapted from Panpanich 2004 \\
\hline \multicolumn{5}{|l|}{ Quality of life } \\
\hline Franke & 2011 & LIC & Adherence & Medical Outcomes Study HIV Health Survey \\
\hline Peltzer & 2010 & $\mathrm{HIC}$ & Adherence & WHOQOL-HIVBREF \\
\hline \multicolumn{5}{|l|}{ Stigma } \\
\hline Rintamaki & 2006 & $\mathrm{HIC}$ & Adherence & 3 items from PMAQ \\
\hline Franke & 2011 & LIC & Adherence & Berger Scale \\
\hline Carlucci & 2011 & LIC & Adherence & Own scale \\
\hline Pettifor & 2004 & $\mathrm{HIC}$ & HIV testing & Genberg scale \\
\hline$\overline{\mathrm{Li}}$ & 2010 & $\mathrm{HIC}$ & Adherence & Adapted from Herek and Capitanio \\
\hline Sayles_a & 2009 & $\mathrm{HIC}$ & Adherence & Own scale \\
\hline Koku & 2011 & LIC & HIV testing & 2003-GDHS \\
\hline \multicolumn{5}{|l|}{ Dicrimination } \\
\hline Boyer & 2011 & LIC & Adherence & Self reported \\
\hline Grierson & 2011 & $\mathrm{HIC}$ & Adherence & Self reported \\
\hline Peltzer & 2010 & $\mathrm{HIC}$ & Adherence & Own 7 items scale \\
\hline Pettifor & 2004 & $\mathrm{HIC}$ & HIV testing & Genberg scale \\
\hline
\end{tabular}


and programs. Indeed, this review showed that according to the quantitative evidence the factors studied to assess the barriers to access HIV/AIDS services inexplicably differ between richer and poorer countries contrary to the evidence from qualitative studies.

Additionally, due to an insufficient number of consistent studies for other outcomes, only adherence outcomes could be meta-analysed. The meta-analysis of the other outcomes proved untenable as the wide range of instruments and indicators used to assess socio-cultural variables such as social support, stigma, depression, and self-efficacy, introduced too much heterogeneity in the studies and impeded the pooling and synthesis of the results. Table 6 shows the variability of the instruments used to assess the same indicator. Risk factors of low-adherence, in both rich and poor countries, were stigma and discrimination, alcohol and drug abuse, depression and low self-efficacy. Social support was the only factor that showed a protective effect. Yet, it is unclear whether this effect occurred equally in rich and poor countries as enough data were available only from high income countries. The comparative approach between high and low income countries of this systematic review and meta-analysis has several advantages over pooling all countries included in the review. This comparative nature yielded valuable information about the differences and similarities of social and cultural processes that affect access to treatment in each context. The comparison also reveals a potential bias in the factors studied in the different regions that may be motivated by cultural stereotypes and has also facilitated the detection of trends and the identification of gaps in the surveys conducted which otherwise would have remained in the shadows. Thus this comparative approach has helped to produce a more detailed description of these gaps which can be beneficial for the preparation of future surveys in this field.

There are several limitations to our study. Publication bias may be limiting our systematic review of quantitative studies although we have used Preferred Reporting Items for Systematic Reviews and Meta-Analyses (PRISMA) guidelines [45] to examine reporting and other biases in a systematic way. See Additional file 1 for further details. Another limitation is the difference between the timing of the preparation of the surveys and the one of the publications of qualitative studies. We would not expect a survey published in the early 90s to be aware of the problems identified in qualitative studies published later. But our analysis indicates that in general the quantitative studies have not systematically addressed important issues identified in qualitative studies that were published at least two years before the implementation of their surveys.

\section{Conclusions}

This review has highlighted a number of issues requiring further research and demonstrated the need to improve the research strategy in epidemiological survey studies. Improvement of this strategy requires better integration of the findings of qualitative studies in quantitative surveys and more consistency between survey studies. This review also offered evidence of the lack of consistency in the measurement of socio-cultural factors which hinders comparisons between studies.

We recommend that, prior to developing a questionnaire, literature reviews should be systematically carried out including qualitative studies. This would help to identify appropriate themes for the context avoiding the tendency to focus on the same topics. We further recommend using validated instruments giving priority to cultural adaptations over the development of new measures. We also call for a generalization of some variables without limiting the specificity of the various contexts. For example, it would be useful to report the effects of different types of stigma, as defined by subscales of validated tools, rather than global scores which cannot be disentangled and are less informative. Another example is the social support measure which could also be broken down by subtypes, material, emotional, etc.

Further quantitative research is needed on socio-cultural determinants of HIV testing, initiation of antiretroviral therapy and defaulting in both low- and high-income countries. More consistency between qualitative and quantitative research and between quantitative measures of socio-cultural factors will help to increase the quality of the data collected, to enhance comparability which is a prerequisite for meta-analyses, to avoid duplication and in general to produce better scientific evidence to inform managers and policy-makers working on HIV/AIDS.

\section{Additional file}

Additional file 1: PRISMA 2009 Checklist.

Competing interests

The authors declare that they have no competing interests.

\section{Authors' contributions}

Conceived and designed the study: SG, SM, JM, AMH. Performed the systematic searches and abstracted data: SG, CD, TS. Analyzed the data: SG, TS. Wrote the paper: SG, SM, AMH, JM, TS, CD. All authors have read and approved the final manuscript.

\section{Acknowledgements}

We are grateful to the Swiss National Science Foundation (SNSF) for the study funding. The funding organization played no role in the search, collection and interpretation of the data, and in the preparation, review, or approval of the manuscript.

\section{Author details}

'Department of Epidemiology and Public Health, Swiss Tropical and Public Health Institute, Basel, Switzerland. 'University of Basel, Basel, Switzerland. ${ }^{3}$ Sophie Davis School of Biomedical Education, City University of New York, New York, USA. ${ }^{4}$ International evaluation consultant, Particip GmBH, Freiburg, Germany. ${ }^{5}$ Department of Population Studies, University of Zambia, Lusaka, Zambia. 
Received: 1 October 2012 Accepted: 22 May 2013

Published: 28 May 2013

\section{References}

1. Nguyen VK, Klot J, Phillips A, Pirkle C: Culture, HIV \& AIDS: An Annotated Bibliography. Paris: UNESCO; 2008.

2. Merten S, Kenter E, McKenzie O, Musheke M, Ntalasha H, Martin-Hilber A: Patient-reported barriers and drivers of adherence to antiretrovirals in sub-Saharan Africa: a meta-ethnography. Trop Med Int Health 2010, 15(1):16-33.

3. Mills EJ, Nachega JB, Bangsberg DR, Singh S, Rachlis B, Wu P, Wilson K, Buchan I, Gill CJ, Cooper C: Adherence to HAART: a systematic review of developed and developing nation patient-reported barriers and facilitators. PLoS Med 2006, 3(11):e438.

4. Ware NC, Idoko J, Kaaya S, Ware NC, Idoko J, Kaaya S, Biraro IA, Wyatt MA, Agbaji O, Chalamilla G, Bangsberg DR: Explaining adherence success in sub- Saharan Africa: an ethnographic study. PLoS Med 2009, 6(1):e11.

5. Deblonde J, De KokerP, Hamers FF, Fontaine J, Luchters S, Temmerman M: Barriers to HIV testing in Europe: a systematic review. Eur J Public Health 2010, 20(4):422-432

6. Obermeyer MC, Osborn M: The utilization of testing and counseling for HIV: a review of the social and behavioral evidence. Am J Public Health 2007, 97(10):1762-1774.

7. Matovu JKB, Makumbi FE: Expanding access to voluntary HIV counseling and testing insub-Saharan Africa: alternative approaches for improving uptake, 2001-2007. Trop Med Int Health 2007, 12(11):1315-1322.

8. World Bank: Country Classification Table. Available: http://data.worldbank.org/ about/country-classifications/country-and-lending-groups K2CKM78CCO 2011

9. Von Elm E, Altman DG, Egger M, Pocock SJ, Gotzsche PC: The strengthening of reporting of observational studies in epidemiology (STROBE) statement guidelines for reporting observational studies. J Clin Epidemiol 2008, 61:344-349.

10. Wells GA, Shea B, O'Connell D, Peterson J, Welch V, Losos M, Tugwell P: The Newcastle-Ottawa Scale (NOS) for Assessing the Quality of Nonrandomised Studies in Meta-analyses. Oxford University Press; 2006. http://www.ohri.ca/ programs/clinical_epidemiology/oxford.asp.

11. Boyer S, Clerc I, Bonono CR, Marcellin F, Bile PC, Ventelou B: Non-adherence to antiretroviral treatment and unplanned treatment interruption among people living with HIV/AIDS in Cameroon: Individual and healthcare supply-related factors. Soc Sci Med 2011, 72(8):1383-1392.

12. Carlucci JG, Kamanga A, Sheneberger R, Shepherd BE, Jenkins CA, Spurrier J, Vermund $\mathrm{SH}$ : Predictors of adherence to antiretroviral therapy in rural Zambia. J Acquir Immune Defic Syndr 2008, 47(5):615-622.

13. Charurat M, Oyegunle M, Benjamin R, Habib A, Eze E, Ele P, Ibanga I, Ajayi S, Eng M, Mondal P, Gebi U, Iwu E, Etiebet MA, Abimiku A, Dakum P, Farley J, Blattner W: Patient retention and adherence to antiretrovirals in a large antiretroviral therapy program in Nigeria: a longitudinal analysis for risk factors. PLoS One 2010, 5(5):e10584.

14. Franke MF, Murray MB, Munoz M, Hernandez-Diaz S, Sebastian JL, Atwood S, Caldas A, Bayona J, Shin SS: Food insufficiency is a risk factor for suboptimal antiretroviral therapy adherence among HIV-infected adults in urban Peru. AIDS Behav 2011, 15(7):1483-1489.

15. Giday A, Shiferaw W: Factors affecting adherence of antiretroviral treatment among AIDS patients in an Ethiopian tertiary university teaching hospital. Ethiop Med J 2010, 48(3):187-194.

16. Karcher H, Omondi A, Odera J, Kunz A, Harms G: Risk factors for treatment denial and loss to follow-up in an antiretroviral treatment cohort in Kenya. Trop Med Int Health 2007, 12(5):687-694.

17. Koku: Desire for, and uptake of HIV tests by ghanaian women: the relevance of community level stigma. J Community Health 2011, 36(2):289-299.

18. Martinez P, Andia I, Emenyonu N, Hahn JA, Hauff E, Pepper L, Bangsberg DR: Alcohol use, depressive symptoms and the receipt of antiretroviral therapy in southwest Uganda. AIDS Behav 2008, 12(4):605-612.

19. Nam NT, Bygbjerg IC, Mogensen HO, Rasch V: Factors associated with the failure to seek HIV care and treatment among HIV-positive women in a Northern Province of Vietnam. AIDS Patient Care STDS 2010, 24(5):325-332.

20. Sarna A, Pujari S, Sengar AK, Garg R, Gupta I, Dam J: Adherence to antiretroviral therapy \& its determinants amongst HIV patients in India. Indian J Med Res 2008, 127(1):28-36.
21. Watt MH, Maman S, Golin CE, Earp JA, Eng E, Bangdiwala SI, Jacobson M: Factors associated with self-reported adherence to antiretroviral therapy in a Tanzanian setting. AIDS Care 2010, 22(3):381-389.

22. Sambisa W, Curtis S, Mishra V: AIDS stigma as an obstacle to uptake of HIV testing: evidence from a Zimbabwean national population-based survey. Aids Care-Psychological and Socio-Medical Aspects of Aids/Hiv 2010, 22(2):170-186

23. Kranzer K, McGrath N, Saul J, Crampin AC, Jahn A, Malema S, Mulawa D, Fine PE, Zaba B, Glynn JR: Individual, household and community factors associated with HIV test refusal in rural Malawi. Trop Med Int Health 2008, 13(11):1341-1350.

24. Aloisi MS, Arici C, Balzano R, Noto P, Piscopo R, Filice G, Menichetti F, d'Arminio Monforte A, Ippolito G, Girardi E, Italian Cohort Naive Antiretrovirals Behavioral Epidemiology Study Group: Behavioral correlates of adherence to antiretroviral therapy. J Acquir Immune Defic Syndr 2002, 31(3):S145-S148.

25. Barfod TS, Gerstoft J, Rodkjaer L, Pedersen C, Nielsen H, Møller A, Kristensen $\mathrm{LH}$, Sørensen HT, Obel N: Patients' answers to simple questions about treatment satisfaction and adherence and depression are associated with failure of HAART: a cross-sectional survey. APCS 2005, 19(5):317-325.

26. Cunningham WE, Andersen RM, Katz MH, Stein MD, Turner BJ, Crystal S, Zierler S, Kuromiya K, Morton SC, St Clair P, Bozzette SA, Shapiro MF: The impact of competing subsistence needs and barriers on access to medical care for persons with human immunodeficiency virus receiving care in the United States. Medical Care 1999, 37(12):1270-1281.

27. de Castilho EA, Szwarcwald CL, de Brito AM: Cofactors of antiretroviral treatment interruption in cases of adults with AIDS: Rio Grande do Norte, Brazil, 1999-2002. Rev Assoc Med Bras 2006, 52(2):86-92.

28. Gebo KA, Keruly J, Moore RD: Association of social stress, illicit drug use, and health beliefs with nonadherence to antiretroviral therapy. $J$ Gen Intern Med 2003, 18(2):104-111.

29. Grierson J, Koelmeyer R, Smith A, Pitts M: Adherence to antiretroviral therapy: factors independently associated with reported difficulty taking antiretroviral therapy in a national sample of HIV-positive Australians. HIV Med 2011, 12(9):562-569.

30. Holmes WC, Bilker WB, Wang H, Chapman J, Gross R: HIV/AIDS-specific quality of life and adherence to antiretroviral therapy over time. J Acquir Immune Defic Syndr 2007, 46(3):323-327.

31. Kranzer K, Lewis JJ, Ford N, Zeinecker J, Orrell C, Lawn SD, Bekker LG, Wood $R$ : Treatment interruption in a primary care antiretroviral therapy program in South Africa: cohort analysis of trends and risk factors. $J$ Acquir Immune Defic Syndr 2010, 55(3):17-23.

32. Li L, Li L, Lee SJ, Wen Y, Lin C, Wan D, Jiraphongsa C: Antiretroviral therapy adherence among patients living with HIV/AIDS in Thailand. Nurs Health Sci 2010, 12(2):212-220.

33. Mugavero M, Ostermann J, Whetten K, Leserman J, Swartz M, Stangl D, Thielman N: Barriers to antiretroviral adherence: the importance of depression, abuse, and other traumatic events. AIDS Patient Care STDS 2006, 20(6):418-428.

34. Okonsky JG: Problems taking pills: understanding HIV medication adherence from a new perspective. AIDS Care 2011, 23(12):1652-1659.

35. Peltzer K, Friend-du Preez N, Ramlagan S, Anderson J: Antiretroviral treatment adherence among HIV patients in KwaZulu-Natal, South Africa. BMC Public Health 2010, 10:111

36. Audrey P, MacPhail C, Sujit S, Delany-Moretlwe: Sinead Factors associated with HIV testing among public sector clinic attendees in Johannesburg, South Africa. Aids and Behavior 2010, 14(4):913-921.

37. Pinheiro CA, De-Carvalho-Leite JC, Drachler ML, Silveira VL: Factors associated with adherence to antiretroviral therapy in HIV/AIDS patients: a cross-sectional study in Southern Brazil. Braz J Med Biol Res 2002, 35(10):1173-1181.

38. Remien RH, Bastos FI, Terto V, Raxach JC, Pinto RM, Parker RG, Berkman A, Hacker MA: Adherence to antiretroviral therapy in a context of universal access, in Rio de Janeiro, Brazil. Aids Care-Psychological and Socio-Medical Aspects of Aids/Hiv 2007, 19(6):740-748.

39. Rintamaki LS, Davis TC, Skripkauskas S, Bennett CL, Wolf MS: Social stigma concerns and HIV medication adherence. AIDS Patient Care STDS 2006, 20(5):359-368.

40. Sayles JN, Wong MD, Kinsler JJ, Martins D, Cunningham WE: The association of stigma with self-reported access to medical care and antiretroviral therapy adherence in persons living with HIV/AIDS. J Gen Intern Med 2009, 24(10):1101-1108. 
41. Sayles JN, Wong MD, Cunningham WE: The inability to take medications openly at home: does it help explain gender disparities in HAART use? J Womens Health (Larchmt) 2006, 15(2):173-181.

42. van Servellen G, Lombardi E: Supportive relationships and medication adherence in HIV-infected, low-income Latinos. West J Nurs Res 2005, 27(8):1023-1039.

43. Wang $X$, Wu Z: Factors associated with adherence to antiretroviral therapy among HIV/AIDS patients in rural China. AIDS 2007, 21(8):S149-S155

44. MacPhail C, Pettifor A, Moyo W, Rees H: Factors associated with HIV testing among sexually active South African youth aged 15-24 years. AIDS Care 2009, 21(4):456-467.

45. Liberati A, Altman DG, Tetzlaff J, Mulrow C, Götzsche PC, loannidis JPA, Clarke M, Devereaux PJ, Kleijnen J, Moher D: The PRISMA statement for reporting systematic reviews and meta-analyses of studies that evaluate healthcare interventions: explanation and elaboration. BMJ 2009, 339:b2700. http://www.bmj.com/content/339/bmj.b2700.

doi:10.1186/1472-6963-13-198

Cite this article as: Gari et al:: Access to HIV/AIDS care: a systematic review of socio-cultural determinants in low and high income countries. BMC Health Services Research 2013 13:198.

\section{Submit your next manuscript to BioMed Central and take full advantage of:}

- Convenient online submission

- Thorough peer review

- No space constraints or color figure charges

- Immediate publication on acceptance

- Inclusion in PubMed, CAS, Scopus and Google Scholar

- Research which is freely available for redistribution 